\title{
Nrf2 mediates the protective effects of homocysteine by increasing the levels of GSH content in HepG2 cells
}

\author{
BING ZHANG $^{1}$, JING-LIN DONG ${ }^{2}$, YING-LI CHEN ${ }^{1}$, YANG LIU ${ }^{1}$, SHI-SHUN HUANG ${ }^{1}$, \\ XIU-LI ZHONG ${ }^{1}$, YU-HONG CHENG ${ }^{3}$ and ZHI-GANG WANG ${ }^{1}$
}

${ }^{1}$ College of Medical Laboratory Science and Technology, Harbin Medical University at Daqing, Daqing, Heilongjiang 163319;

${ }^{2}$ Daqing Oilfield General Hospital, Daqing, Heilongjiang 163001; ${ }^{3}$ Inspection Department,

Daqing Medical College, Daqing, Heilongjiang 163311, P.R. China

Received April 8, 2016; Accepted March 14, 2017

DOI: $10.3892 / \mathrm{mmr} .2017 .6633$

\begin{abstract}
Homocysteine (Hcy) and glutathione (GSH) are crucial reduction-oxidation mediators. The underlying mechanisms governing the effects of Hcy on GSH generation in the progression of alcoholic liver disease has so far received little attention. The present study hypothesized that the antioxidant transcriptional factor nuclear factor (erythroid-derived 2)-like 2 (Nrf2) may participate in Hcy-mediated regulation of GSH production in HepG2 human liver cancer cells. MTT assay was used to study the cytotoxicity of homocysteine, western blot analysis and immunofluorescence staining were used to determine the effect of Hcy on Nrf2 expression. Our data demonstrated that HepG2 cells exposed to exogenous levels of Hcy $(0-100 \mu \mathrm{M})$ exhibited elevated GSH levels in a concentration-dependent manner. Furthermore, 4-hydroxynonenal (4-HNE)-induced cell injury was attenuated by Hcy; however, this protective effect was blocked by the GSH-production inhibitor buthionine sulfoximine. Hcy treatment was able to induce Nrf2 protein expression in HepG2 cells. Treatment with the Nrf2 activator tert-butylhydroquinone $(0-100 \mu \mathrm{M})$ increased GSH expression in a concentration-dependent manner; however, Nrf2-siRNA abolished the Hcy-induced increase in GSH expression and cellular protection in 4-HNE-stressed HepG2 cells. In conclusion, the antioxidant transcriptional factor Nrf2 was demonstrated to mediate the Hcy-induced increase in GSH expression levels and cellular protection in HepG2 cells.
\end{abstract}

\footnotetext{
Correspondence to: Professor Zhi-Gang Wang, College of Medical Laboratory Science and Technology, Harbin Medical University at Daqing, 39 Xinyang Road, Daqing, Heilongjiang 163319, P.R. China E-mail: 1007223943@qq.com
}

Key words: nuclear factor (erythroid-derived 2)-like 2, homocysteine, glutathione, 4-hydroxynonenal, oxidative stress

\section{Introduction}

Homocysteine (Hcy) is a sulfur-containing, non-protein amino acid that is derived from methionine metabolism (1). An imbalance in Hcy metabolism is considered an independent risk factor for many disorders, such as physical impairment (2), atherosclerosis $(3,4)$, neurodegenerative diseases (5) and alcoholic liver disease (ALD) (6,7). Alcoholic hyperhomocysteinemia (HHcy) is often seen in rodents and humans with during the development of alcoholic steatohepatitis (8). In contrast with the adverse effects of Hcy in the progression of ALD, Hcy may serve a positive role in cellular antioxidant reactions as an important producer of cellular total glutathione (GSH) $(9,10)$. This metabolic link between Hcy and GSH occurs by the transsulfuration of Hcy to cysteine, a precursor for GSH $(11,12)$. GSH performs a crucial role in maintaining hepatic reduction-oxidation (redox) balance in biological systems (13). However, the underlying molecular mechanism of the positive role that Hcy serves in the progression of ALD has not been clearly elucidated.

The nuclear factor (erythroid-derived 2)-like 2 (Nrf2) functions as a master regulator of the antioxidant response by regulating the expression of cytoprotective genes, including genes involved in the antioxidant GSH pathway (14-16). Notably, a Hcy-activated Nrf2-antioxidant reaction has been demonstrated in mouse macrophage and human hepatoma cell lines $(6,9,10)$. Therefore, the present study hypothesized that Nrf2 may function to mediate Hcy-regulated GSH production in hepatocytes, and that the hepatic Hcy-Nrf2-GSH pathway may serve a protective role in the progression of ALD. Cellular viability and intracellular GSH expression levels were assayed to investigate the antioxidant effects of Hcy and Nrf2 on HepG2 cell injury induced by the hepatic lipid-peroxidation product 4-hydroxynonenal (4-HNE). Hcy-activated Nrf2 expression was demonstrated by western blot analysis and immunofluorescence staining assay. Further research into this area will provide deeper insights into the association between Hcy and its potentially protective roles in the progression of ALD.

\section{Materials and methods}

Cell culture conditions and MTT assay. The HepG2 human hepatocarcinoma cell line was purchased from the American 
Type Culture Collection (Manassas, VA, USA) and maintained in Dulbecco's modified Eagle's medium (DMEM) containing $10 \%$ fetal bovine serum (FBS; both from HyClone; GE Healthcare Life Sciences, Logan, UT, USA) at $37^{\circ} \mathrm{C}$ in a $5 \% \mathrm{CO}_{2}$ atmosphere.

Immunofluorescence staining. HepG2 cells were grown on 6 -well cover slips at a density of $3 \times 10^{3}$ cells/well, and treated with Hcy $(0,10,20,50$ or $100 \mu \mathrm{M})$ for $24 \mathrm{~h}$ at $37^{\circ} \mathrm{C}$. Adherent cells were fixed to slides with paraformaldehyde for $15 \mathrm{~min}$ at $37^{\circ} \mathrm{C}$ and incubated with $0.5 \%$ Trion-X-100 for $10 \mathrm{~min}$ at $37^{\circ} \mathrm{C}$. Nonspecific binding was blocked by $3 \%$ BSA for $30 \mathrm{~min}$ at $37^{\circ} \mathrm{C}$ as previously described (17). Immunostaining was performed with an anti-Nrf2 antibody overnight at $4^{\circ} \mathrm{C}(1: 300$; bs-1074R, Rabbit polyclonal IgG, BIOSS, Beijing, China), followed by incubation with a cyanine-3 (Cy3)-conjugated secondary antibody for $2 \mathrm{~h}$ at $37^{\circ} \mathrm{C}(1: 200$. P0183, Goat Anti-Rabbit $\mathrm{IgG}$, Beyotime Institute of Biotechnology, Shanghai, China). Nuclear staining was performed by incubating with DAPI (1:100) for $10 \mathrm{~min}$ at $37^{\circ} \mathrm{C}$. Increased Nrf2 expression was observed under an inverted microscope (Nikon Corporation, Tokyo, Japan). Images were processed using Image ProPlus 6.0 (Media Cybernetics, Inc., Rockville, MD, USA).

Knockdown of Nrf2 by siRNA. Nrf2 expression was silenced by Nrf2-siRNA (Suzhou GenePharma LLC). A non-targeted control siRNA (NC) was used as a negative control. The siRNA sense sequences were as follows: siNrf2, 5'-GCACCUUAU AUCUCGAAGUTT-3'; NC, 5'-UUCUCCGAACGUGUCACG UTT-3'. Briefly, HepG2 cells were grown to $50-70 \%$ confluence. A total of $0.27 \mu \mathrm{g}(1 \mu \mathrm{l})$ siRNA, $10 \mu \mathrm{l}$ INTERFERin Gene siRNA Transfection Reagent (Suzhou GenePharma LLC) and $400 \mu 1$ serum-free Opti-MEM-1 medium (Gibco; Thermo Fisher Scientific, Inc., Waltham, MD, USA) were combined and added to the cells, which were incubated at room temperature for $10 \mathrm{~min}$, followed by the subsequent addition of the siRNA/Transfection Reagent mixture directly onto the cells with $4 \mathrm{ml}$ serum-free Opti-MEM-1 medium (final siRNA concentration, $1 \mathrm{nM}$ ). Following incubation for $4 \mathrm{~h}$ at $37^{\circ} \mathrm{C}$, the media was replaced with DMEM containing 10\% FBS. Total RNA was extracted and reverse transcribed to cDNA using a Golden 1st cDNA Synthesis kit (HaiGene Bio Inc., Harbin, China). cDNA was stored at $-80^{\circ} \mathrm{C}$ and transfection efficiency was later validated by reverse transcription-quantitative polymerase chain reaction (RT-qPCR).

MTT assay. HepG2 cells were seeded at a density of $2 \times 10^{3}$ cells/well in two 96-well plates; one plate was treated with $70 \mu \mathrm{M}$ 4-HNE (Cayman Chemical Company, Ann Arbor, MI, USA), $200 \mu \mathrm{M}$ buthionine sulfoximine (BSO; Shanghai Aladdin Bio-Chem Technology Co., Ltd., Shanghai, China), $100 \mu \mathrm{M}$ Hcy (Sigma-Aldrich; Merck KGaA, Darmstadt, Germany), 4-HNE+Hcy, BSO+Hcy, BSO+4-HNE and $\mathrm{BSO}+4-\mathrm{HNE}+\mathrm{Hcy}$ for $24 \mathrm{~h}$ at $37^{\circ} \mathrm{C}$; the second plate was transfected with $1 \mathrm{nM}$ negative control and $\mathrm{Nrf} 2$-targeted small interfering RNA (siRNA; Suzhou GenePharma LLC, Suzhou, China), followed by addition of $100 \mu \mathrm{M} \mathrm{Hcy,} 70 \mu \mathrm{M} 4$-HNE or 4-HNE+Hcy respectively, for $24 \mathrm{~h}$ at $37^{\circ} \mathrm{C}$. The following day, $20 \mu 1$ MTT $(5 \mathrm{mg} / \mathrm{ml})$, the yellow mitochondrial dye, was added to the cells, and the plates were incubated at $37^{\circ} \mathrm{C}$ for an additional $4 \mathrm{~h}$. The culture medium was discarded, and any formazan crystals that may have formed were dissolved in $150 \mu \mathrm{l}$ DMSO. Absorbance was measured at $570 \mathrm{~nm}$ using a SPECTRA Max 190 (Molecular Devices, LLC, Sunnyvale, CA, USA).

Measurement of reduced GSH and oxidized GSH levels in Hep $\mathrm{G} 2$ cells. HepG2 cells $\left(3 \times 10^{5}\right)$ were treated with increasing concentrations of Hcy $(0-500 \mu \mathrm{M})$ and tert-butylhydroquinone (t-BHQ; 0-100 $\mu \mathrm{M}$, Sigma-Aldrich; Merck KGaA) and $200 \mu \mathrm{M}$ BSO for $24 \mathrm{~h}$, respectively, or transfected with $1 \mathrm{nM}$ Nrf2- or $\mathrm{NC}$-siRNA for $4 \mathrm{~h}$ at $37^{\circ} \mathrm{C}$. The media was subsequently replaced with DMEM containing 10\% FBS with or without Hcy for $9 \mathrm{~h}$. Cells were trypsinized and collected by centrifugation at $170 \mathrm{x} \mathrm{g}$ for $5 \mathrm{~min}$ at room temperature, re-suspended in protein removal reagent $\mathrm{M}$ (Beyotime Institute of Biotechnology) and immediately vortex mixed for $10 \mathrm{~s}$. The samples were frozen rapidly with liquid nitrogen and thawed at $37^{\circ} \mathrm{C}$, this was repeated twice, and then the samples were incubated in an ice-bath for $5 \mathrm{~min}$. The resulting supernatant was collected by centrifugation at $10,000 \mathrm{x}$ g for $10 \mathrm{~min}$, and analyzed using the sulfhydryl reagent 5,5'-dithiobis (2-nitrobenzoic acid), which forms the yellow derivative 5'-thio-2-nitrobenzoic acid (TNB), and is measured at $412 \mathrm{~nm}$ using a SPECTRA Max 190 (Molecular Devices, LLC). Levels of reduced and oxidized GSH were determined using a GSH Assay kit (Beyotime Institute of Biotechnology), according to the manufacturers protocol (18).

Western blot analysis. HepG2 cells were treated with Hcy $(0-100 \mu \mathrm{M})$ for $24 \mathrm{~h}$ at $37^{\circ} \mathrm{C}$. Cells were homogenized in RIPA lysis buffer (Beyotime Institute of Biotechnology) containing leupeptin. Protein concentrations were quantified using a Bicinchoninic Acid Protein Assay kit (Beyotime Institute of Biotechnology), according to the manufacturer's protocol. Cellular protein samples $(30 \mu \mathrm{g})$ were separated by $10 \%$ SDS-PAGE as previously described (19). Proteins were transferred onto nitrocellulose membranes and the membranes were incubated with an anti-Nrf2 polyclonal antibody (1:500; bs-1074R, BIOSS, Beijing, China) or an anti- $\beta$-actin monoclonal antibody (1:3,000; TA-09; Zhongshan Golden Bridge Biotechnology, Beijing, China) overnight at $4^{\circ} \mathrm{C}$, followed by horseradish peroxidase-conjugated goat anti-rabbit immunoglobulin G secondary antibody (1: 5,000; ZB-2301, Zhongshan Golden Bridge Biotechnology). Protein bands were visualized by enhanced chemiluminescence (HaiGene Bio Inc.). Densitometric analysis of band intensity was calculated using Quantity One software, version v4.62 (Bio-Rad Laboratories, Inc., Hercules, CA, USA).

Relative $m R N A$ expression quantification by RT-qPCR. Total RNA was extracted from cultured HepG2 cells $\left(2 \times 10^{5}\right.$ cells $)$ using TRIzol Reagent (HaiGene Bio Inc.), according to the manufacturer's protocol (9), and subsequently reverse transcribed to cDNA using a Golden 1st cDNA Synthesis Kit (HaiGene Bio Inc.). cDNA samples were amplified using a DNA thermal cycler (Thermo Fisher Scientific, Inc.). qPCR reactions were set up by mixing 10X SYBR Green PCR Master Mix (Roche Diagnostics GmbH, Mannheim, Germany) with $2 \mu \mathrm{l} 100$ fold diluted cDNA template, $0.6 \mu \mathrm{l}$ each of forward 
and reverse primers $(0.3 \mu \mathrm{M})$ and $6.8 \mu \mathrm{l}$ PCR-grade water in a $20 \mu \mathrm{l}$ reaction volume. The reaction mixtures were amplified using an ABI 7300 Sequence Detection System (Applied Biosystems; Thermo Fisher Scientific, Inc.) with an initial denaturation step at $95^{\circ} \mathrm{C}$ for $10 \mathrm{~min}$, followed by 40 cycles of: denaturation at $95^{\circ} \mathrm{C}$ for $15 \mathrm{sec}$; annealing at $58^{\circ} \mathrm{C}$ for $20 \mathrm{sec}$; extension at $72^{\circ} \mathrm{C}$ for $30 \mathrm{sec}$; and melting curve analysis. The primer sequences were as follows: $18 \mathrm{~S}$, forward $5^{\prime}-\mathrm{AAC}$ TTTCGATGGTAGTCGCCG-3', reverse 5'-CCTTGGATG TGGTAGCCGTTT-3'; Nrf2, forward 5'-GCACCUUAUAUC UCGAAGUTT-3', reverse 5'-ACUUCGAGAUAUAAGGUG CTT-3'; GCLc, forward 5'-ATGTGGACACCCGATGCA GTATT-3'; reverse 5'-TGTCTTGCTTGTAGTCAGGATGGT TT-3'. Primers were designed using the GenBank database (https://www.ncbi.nlm.nih.gov/genbank). Nrf2 mRNA and GCLc mRNA expression were measured by RT-qPCR and normalized to 18s. Data was analyzed using Applied Biosystems SDS V1.4 software (Thermo Fisher Scientific, Inc.) using the $2^{-\Delta \Delta \mathrm{Cq}}$ method, where $\Delta \Delta \mathrm{Cq}=\Delta \mathrm{Cq}$ target $-\Delta \mathrm{Cq}$ internal control (20).

Statistical analysis. Statistical analysis was performed using one-way analysis of variance followed by Newman-keuls test. Analyses were performed using OriginPro7.5 software (OriginLab, Northampton, MA, USA) and Graph Pad Prism version 5 (Graph Pad Software Inc., La Jolla, CA, USA). Data were presented as the mean \pm standard error of the mean. Values of $\mathrm{P}<0.05$ were considered to indicate a statistically significant difference. All experiments were performed at least three times and representative results were presented.

\section{Results}

Hcy treatment increases total GSH expression levels in HepG2 cells. Intracellular levels of the antioxidant thiol GSH were evaluated using the GSH Assay kit. In response to exogenous Hcy $(10,20,100$ or $500 \mu \mathrm{M})$ treatment, the levels of intracellular total GSH (Fig. 1A), oxidized GSH (Fig. 1B) and reduced GSH (Fig. 1C) were increased in a concentration-dependent manner, with a peak at $100 \mu \mathrm{M}$ Hcy. Therefore, exogenous Hcy was administered at a concentration of $100 \mu \mathrm{M}$ for the following tests.

Hcy protects against oxidative stress-induced cell injury in HepG2 cells. HepG2 cells were pretreated with the specific $\mathrm{GSH}$ production inhibitor BSO $(200 \mu \mathrm{M})$ for $2 \mathrm{~h}$, and subsequently treated or co-treated with Hcy $(100 \mu \mathrm{M})$ for $24 \mathrm{~h}$. Hcy treatment led to elevated GSH content compared with the untreated control group $(\mathrm{n}=6, \mathrm{P}<0.05)$. However, BSO co-treatment significantly abolished the Hcy-induced GSH expression in HepG2 cells, compared with the Hcy-treated group (Fig. 2A; $\mathrm{n}=6 ; \mathrm{P}<0.05)$. HepG2 cells were treated with several combinations, as follows: 4-HNE $(70 \mu \mathrm{M})+\mathrm{Hcy}(100 \mu \mathrm{M})$; BSO $(200 \mu \mathrm{M})+4-\mathrm{HNE}$; BSO+Hcy; $\mathrm{BSO}+4-\mathrm{HNE}+\mathrm{Hcy}$ all together. MTT assay indicated that treatment with the lipid peroxidation product 4-HNE decreased cell viability, and no cellular toxicity was observed in HepG2 cells at $10 \mu \mathrm{M}$ Hcy. However, Hcy co-treatment attenuated 4-HNE-induced cell injury, compared with the 4-HNE-treated group (Fig. 2B; $n=6$; $\mathrm{P}<0.05)$. Notably, BSO pretreatment significantly weakened

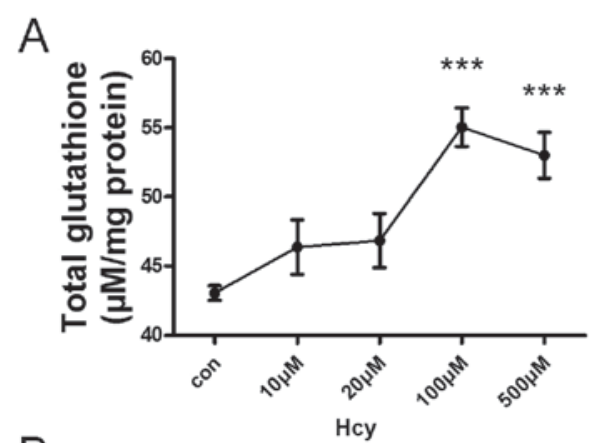

B
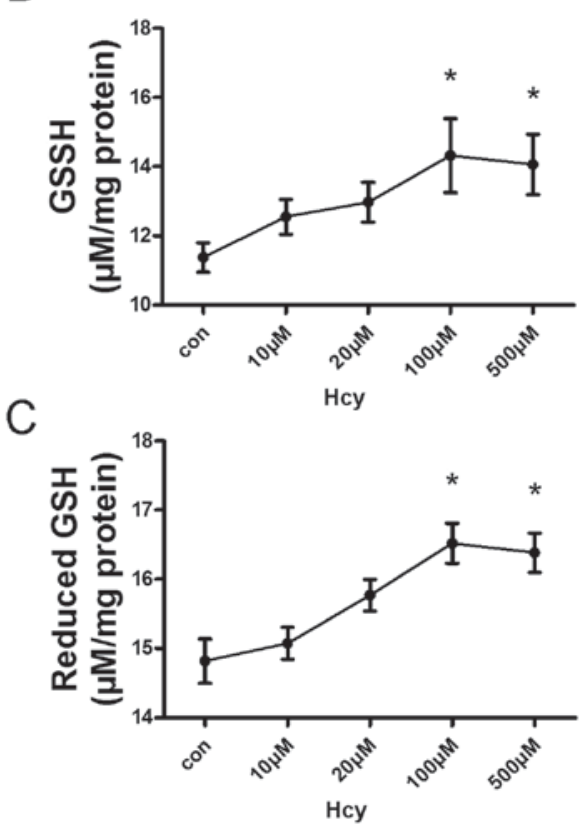

Figure 1. GSH levels in response to Hcy treatment in HepG2 cells. HepG2 cells were treated with Hcy $(0-500 \mu \mathrm{M})$ for $24 \mathrm{~h}$. The levels of (A) total GSH, (B) oxidized GSH and (C) reduced GSH were determined using a GSH Assay kit. Values are presented as the mean \pm standard error of the mean $(n=3)$. ${ }^{*} \mathrm{P}<0.05,{ }^{* * *} \mathrm{P}<0.001 ; \mathrm{GSH}$, total glutathione; Hcy, homocysteine.

the protective effect of Hcy on 4-HNE-induced cell injury in HepG2 cells, compared with the 4-HNE+Hcy group $(\mathrm{n}=6 ; \mathrm{P}<0.05)$. Conversely, BSO pretreatment had no effect on viability in Hcy- or 4-HNE-treated cells (Fig. 2B; $n=6$; $\mathrm{P}>0.05)$.

Hcy treatment induces Nrf 2 protein expression and promotes nuclear accumulation in HepG2 cells. HepG2 cells were treated with Hcy $(0-100 \mu \mathrm{M})$ for $24 \mathrm{~h}$. Western blot analysis revealed that Hcy exposure induced $\mathrm{Nrf} 2$ protein expression in a concentration-dependent manner (Fig. 3A). Immunofluorescence analysis with an anti-Nrf2 antibody revealed that the protein expression of Nrf2 increased with the Hcy concentration (Fig. 3B). In addition, Hcy $(0-100 \mu \mathrm{M})$ treatment promoted the expression of glutamate-cysteine ligase catalytic subunit (GCLc), a downstream target gene of Nrf2 (Fig. 3C).

Nrf2 mediates Hcy-induced GSH expression and cell protection. To explore whether Nrf2 participates in the Hcy-induced increase in GSH expression in HepG2 cells, cells were treated with the Nrf2 activator t-BHQ $(0,10,20,50$ or $100 \mu \mathrm{M})$ for $24 \mathrm{~h}$. 
A

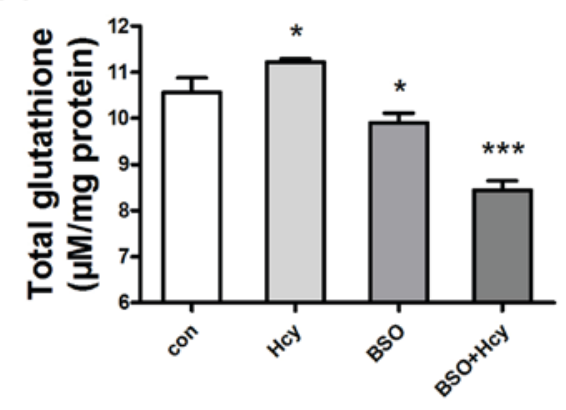

B

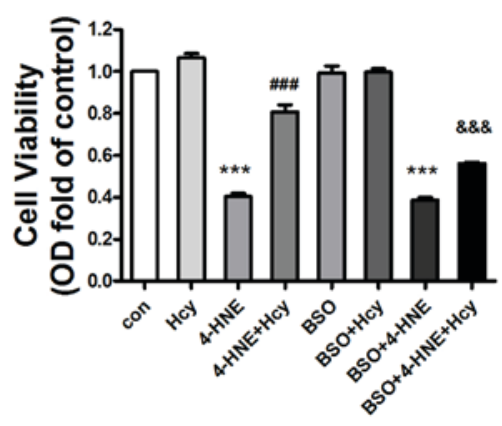

Figure 2. Hcy treatment protects HepG2 cells from 4-HNE-induced cell injury by increasing GSH expression levels. (A) Treatment with the GSH biosynthesis inhibitor BSO abolished Hcy-induced GSH expression in HepG 2 cells. HepG2 cells were pretreated with or without $200 \mu \mathrm{M}$ BSO for 2 h, followed by treatment with $100 \mu \mathrm{M}$ Hcy for $24 \mathrm{~h}$. Total GSH levels were determined using a GSH Assay kit. (B) Hcy treatment reduced 4-HNE-induced cell injury, and co-treatment with BSO weakened the protective effect of Hcy on 4-HNE-induced cell injury in HepG2 cells. HepG2 cells were pretreated with or without BSO for 2 h, followed by treatment with $100 \mu \mathrm{M}$ Hcy and/or $70 \mu \mathrm{M} 4$-HNE for $24 \mathrm{~h}$. The viability of HepG2 was detected by MTT assay. Values are presented as the mean \pm standard error of the mean $(\mathrm{n}=6)$. ${ }^{*} \mathrm{P}<0.05$ and ${ }^{* * *} \mathrm{P}<0.001$ vs. control or Hcy group; ${ }^{\# \#} \mathrm{P}<0.001$ vs. 4-HNE group; \&\&\& $\mathrm{P}<0.001$ vs. 4-HNE+Hcy group. 4-HNE, 4-hydroxynonenal; BSO, buthionine sulfoximine; Con, control; GSH, total glutathione; Hcy, homocysteine.

Cells exposed to t-BHQ exhibited an increase in GSH expression levels in a concentration-dependent manner (Fig. 4A). Additionally, Nrf2 gene expression was knocked down in HepG2 cells by siRNA, which resulted in the decreased expression of Nrf2 mRNA levels by $\sim 50 \%$ (Fig. 4B; $n=3 ; \mathrm{P}<0.001$ vs. $\mathrm{NC}$ treated cells). Hcy+NC treatment for 24 h slightly elevated the intracellular GSH content in comparison with the NC group. This small increase may be attributed to invasion of the exogenous siRNA into the serum-starved cells, which may have resulted in decreasing the cell sensitivity to the following Hcy treatment. Nrf2-siRNA exposure resulted in decreased GSH expression levels, compared with the NC-treated HepG2 cells. Furthermore, Hcy-induced GSH expression in HepG2 cells was also suppressed by Nrf2 siRNA, compared with the $\mathrm{NC}+\mathrm{Hcy}$ group (Fig. $4 \mathrm{C} ; \mathrm{n}=3 ; \mathrm{P}<0.05$ ). Analysis of cell viability with MTT indicated that Nrf2-siRNA pretreatment reduced the protective role of Hcy in limiting 4-HNE-induced cell injury in HepG2 cells (Fig. 4D; $n=3 ; \mathrm{P}<0.05$ ). In brief, Nrf2 was observed to mediate Hcy-induced GSH expression and the protective effect of Hcy on cell viability.

\section{Discussion}

The present study is the first, to the best of our knowledge, to demonstrate the protective role of exogenous Hcy in 4-HNE-induced cell injury in HepG2 cells. Furthermore, the present study investigated the underlying molecular mechanism of this process, which involves an increase in GSH expression mediated by the antioxidant transcriptional factor Nrf2.

Previous studies indicated that circulating and hepatic Hcy levels were elevated in alcohol-fed mice, and aberrant Hcy metabolism elicited oxidative stress and hepatic GSH depletion, which has been demonstrated in both experimental animals and in humans $(21,22)$. One study indicated that Hcy treatment was able to trigger acute GSH depletion in HepG2 cells within 45 min (6). By contrast, increased GSH expression that was induced by Hcy has also been demonstrated in HepG2 cells (9) and macrophages (10); this may be due to increased activity via the transsulfuration pathway or de novo synthesis of GSH. Consistent with these reports, the present study demonstrated that $24 \mathrm{~h} \mathrm{Hcy}$ treatment increased the levels of intracellular GSH expression in a concentration-dependent manner. GSH is a crucial mediator in various antioxidant responses; therefore, the data suggested that the GSH metabolism-related amino acid Hcy may be involved in the antioxidant process, and may protect against oxidative stress-induced cell injury in HepG2 cells.

HHcy is a risk factor for cardiovascular disease and ALD, and patients with HHcy may develop hepatic steatosis and fibrosis $(1,3,23,24)$. However, the balance of Hcy metabolism and its role in the progression of these diseases is not clearly understood. In contrast to the adverse effects of elevated Hcy in these pathological conditions, the present study indicated that exogenous Hcy treatment may protect HepG2 cells against cell injury induced by the lipid peroxidation product 4-HNE. Notably, a previous in vitro investigation indicated that exogenous Hcy exposure had no effect on HepG2 cell viability (6). Consistent with this observation, the present study demonstrated that Hcy exposure did not affect HepG2 cell viability in non-stress conditions. Also of note, the GSH biosynthesis inhibitor BSO significantly lowered the Hcy-induced increase in GSH expression levels and the Hcy-mediated cellular protection against 4-HNE. Collectively, these data indicated that Hcy protects against 4-HNE-indued cell injury in HepG2 cells, via increased expression of intracellular GSH levels.

Nrf2 is critical for maintaining the GSH redox state and protecting cells against oxidative stress (19). High levels of Hcy treatment suppressed Nrf2-dependent antioxidant protection, but lower levels of Hcy $(100 \mu \mathrm{M})$ upregulated the expression of Nrf2 in U373 astroglial cells (25). In addition, HepG2 cells exposed to $50 \mu \mathrm{M}$ Hcy exhibited an increase in Nrf2 protein expression in a time-dependent manner (9). In the present study, HepG2 cells exposed to Hcy $(0-100 \mu \mathrm{M})$ for $24 \mathrm{~h}$ exhibited increased Nrf2 protein expression in a concentration-dependent manner. These results indicated that 

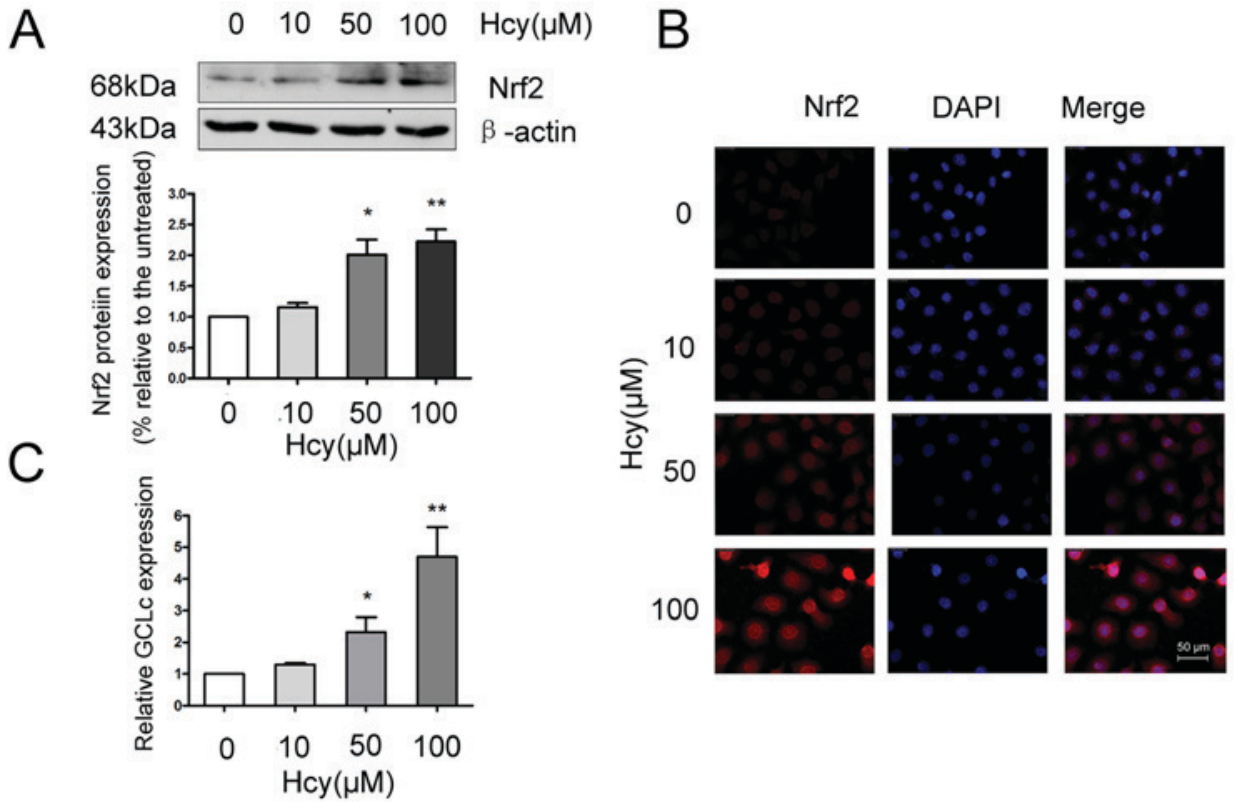

Figure 3. Hcy induces Nrf2 protein expression. HepG2 cells were exposed to Hcy (0-100 $\mu \mathrm{M})$ for $24 \mathrm{~h}$. (A) Hcy treatment induced Nrf2 protein expression in a concentration-dependent manner. Nrf2 expression was assessed by western blot analysis; a representative western blot and semi-quantification are presented. (B) Hcy treatment promoted Nrf2 protein nuclear accumulation in HepG2 cells. Scale bar, $50 \mu \mathrm{m}$. (C) Hcy (0-100 $\mu \mathrm{M})$ treatment increased GCLc expression levels in a concentration-dependent manner. Values are presented as the mean \pm standard error of the mean $(\mathrm{n}=3)$. ${ }^{*} \mathrm{P}<0.05$ and ${ }^{* *} \mathrm{P}<0.01$ vs. untreated $(0 \mu \mathrm{M})$ control. GCLc, glutamate cysteine ligase catalytic subunit; Hcy, homocysteine; Nrf2, nuclear factor erythroid-derived 2-like 2.

A

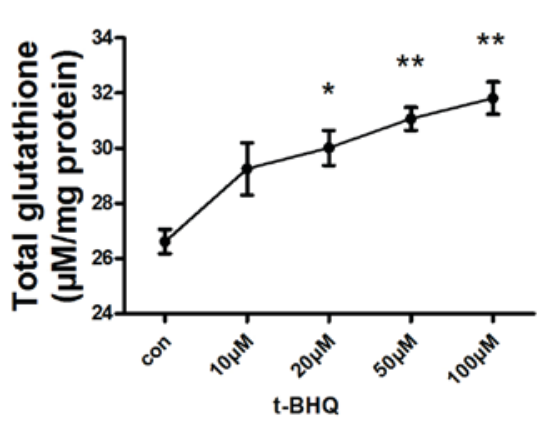

C

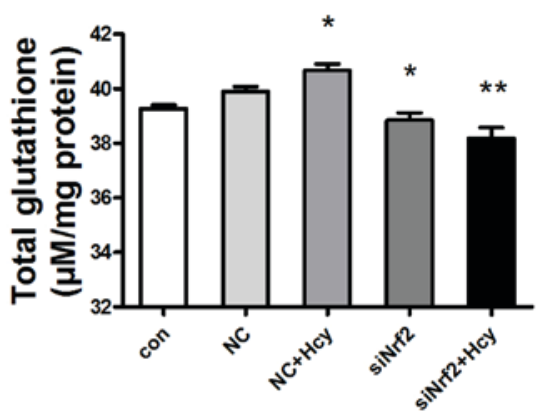

B

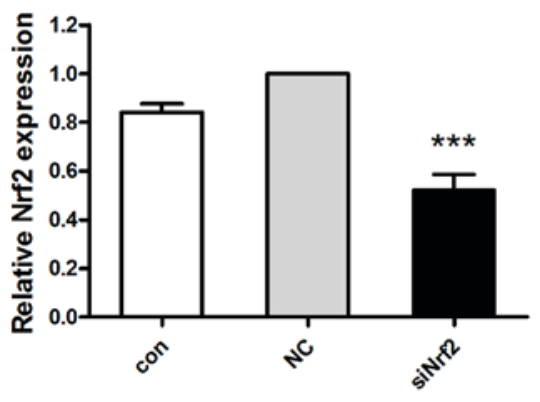

$\mathrm{D}$

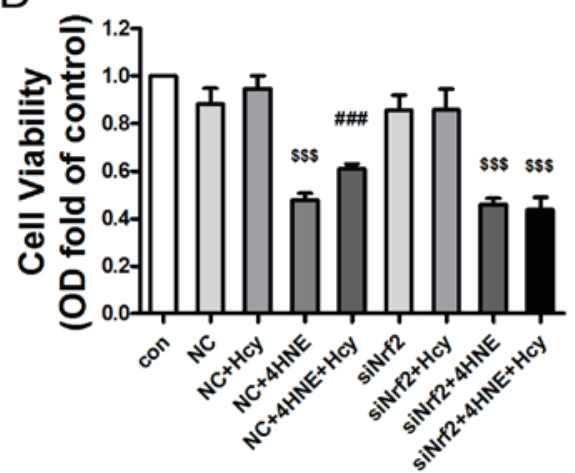

Figure 4. Nrf2 mediates Hcy-induced GSH expression and cellular protection in 4-HNE-stressed HepG2 cells. (A) t-BHQ treatment increased total GSH expression levels in a concentration-dependent manner in HepG2 cells. HepG2 cells were treated with t-BHQ $(0-100 \mu \mathrm{M})$ for $24 \mathrm{~h}(\mathrm{n}=3)$. (B) Nrf2 mRNA expression was downregulated by $\sim 50 \%$ following transfection with Nrf2-siRNA. Nrf2 siRNA transfection was performed for $4 \mathrm{~h}$; the transfection media was subsequently removed and replaced with medium containing $10 \%$ fetal bovine serum for $9 \mathrm{~h}(\mathrm{n}=3)$. (C) Nrf2-siRNA transfection reduced Hcy-induced total GSH elevation. HepG2 cells were transfected with NC-siRNA or Nrf2-siRNA for $4 \mathrm{~h}$, then co-treated with Hcy for $9 \mathrm{~h}$ ( $\mathrm{n}=3$ ). (D) Nrf2 siRNA attenuated the protective role of Hcy in 4-HNE-induced cell injury in HepG2 cells. HepG2 cells were pretreated with $1 \mathrm{nM} \mathrm{NC}$ siRNA or $1 \mathrm{nM}$ Nrf2 siRNA for $4 \mathrm{~h}$, followed by Hcy $(100 \mu \mathrm{M})$ and/or 4-HNE $(70 \mu \mathrm{M})$ for $24 \mathrm{~h}$. Cellular viability was assessed by MTT Assay $(\mathrm{n}=6)$. Values are presented as the mean \pm standard error of the mean. ${ }^{\prime} \mathrm{P}<0.05,{ }^{* *} \mathrm{P}<0.01$ and ${ }^{* * * *} \mathrm{P}<0.001$ vs. control group; ${ }^{\$ \$ \$} \mathrm{P}<0.001$ vs. NC group; ${ }^{\# \# \#} \mathrm{P}<0.001$ vs. NC+4-HNE-treated cells. 4-HNE, 4-hydroxynonenal; Con, control; GSH, glutathione; Hcy, homocysteine; NC, non-targeted control siRNA; Nrf2, nuclear factor erythroid-derived 2-like 2; siRNA, small interfering RNA; t-BHQ, tert-butylhydroquinone 
nontoxic concentrations of Hcy may activate the antioxidant transcriptional factor Nrf2 in HepG2 cells.

Previous studies have reported that Nrf2 affects GSH homeostasis by regulating de novo synthesis and salvage pathways, to protect cells against oxidative stress $(15,16,25,26)$. Consistent with these reports, the present study demonstrated that the Nrf2 activator t-BHQ increased total intracellular GSH levels in a concentration-dependent manner. By contrast, Nrf2-siRNA treatment inhibited the Hcy-induced GSH elevation. Furthermore, Nrf2-siRNA treatment reduced the ability of Hcy to protect against 4-HNE-induced cell injury. Collectively, these data suggested that Nrf2 mediates Hcy-induced GSH expression and cellular protection in 4-HNE-treated HepG2 cells.

In conclusion, the present study demonstrated that Hcy treatment may protect HepG2 cells against cell injury induced by the lipid peroxidation product $4-\mathrm{HNE}$, by inducing the expression of GSH, and this was mediated by the antioxidant transcriptional factor Nrf2. Hcy has been regarded as an independent risk factor for the progression of arteriosclerosis, hypertension or ALD $(3,24,27,28)$. The concentration of Hcy $(<100 \mu \mathrm{M})$ applied in the present study represented mild moderate HHcy, which is usually a characteristic symptom in the early stages of these diseases; an initial elevation of Hcy may serve a compensatory protective effect on vulnerable cells. Therefore, the results indicated that hepatic Hcy elevation may be involved in an antioxidant mechanism, particularly in the early stage of these pathological conditions.

\section{Acknowledgements}

This work was supported by The National Natural Science Foundation (grant no. 81370523), The Postdoctoral Science Foundation Special Project (grant no. 201104420), The China Postdoctoral Science Foundation General Project (grant no. 20100471022) and The Heilongjiang Young Key Academic Staff support program (grant no. 1251G039).

\section{References}

1. Ganguly P and Alam SF: Role of homocysteine in the development of cardiovascular disease. Nutr J 14: 6, 2015.

2. Veeranki S and Tyagi SC: Defective homocysteine metabolism: Potential implications for skeletal muscle malfunction. Int J Mol Sci 14: 15074-15091, 2013.

3. Zhang D, Fang P, Jiang X, Nelson J, Moore JK, Kruger WD, Berretta RM, Houser SR, Yang X and Wang H: Severe hyperhomocysteinemia promotes bone marrow-derived and resident inflammatory monocyte differentiation and atherosclerosis in LDLr/CBS-deficient mice. Circ Res 111: 37-49, 2012.

4. Kamat PK, Vacek JC, Kalani A and Tyagi N: Homocysteine induced cerebrovascular dysfunction: A link to alzheimer's disease etiology. Open Neurol J 9: 9-14, 2015.

5. Sharma M, Tiwari M and Tiwari RK: Hyperhomocysteinemia: Impact on neurodegenerative diseases. Basic Clin Pharmacol Toxicol 117: 287-296, 2015

6. Mani M, Golmohammadi T, Khaghani S, Zamani Z, Azadmanesh K, Meshkani R and Pasalar P: Homocysteine induces heme oxygenase-1 expression via transcription factor Nrf2 activation in HepG2 cell. Iran Biomed J 17: 93-100, 2013.

7. Ji C: Mechanisms of alcohol-induced endoplasmic reticulum stress and organ injuries. Biochem Res Int 2012: 216450, 2012.

8. Esfandiari F, Medici V, Wong DH, Jose S, Dolatshahi M, Quinlivan E, Dayal S, Lentz SR, Tsukamoto $\mathrm{H}$, Zhang $\mathrm{YH}$, et al: Epigenetic regulation of hepatic endoplasmic reticulum stress pathways in the ethanol-fed cystathionine beta synthase-deficient mouse. Hepatology 51: 932-941, 2010.
9. Mani M, Khaghani S, Gol Mohammadi T, Zamani Z, Azadmanesh K, Meshkani R, Pasalar P and Mostafavi E: Activation of Nrf2-antioxidant response element mediated glutamate cysteine ligase expression in hepatoma cell line by homocysteine. Hepat Mon 13: e8394, 2013.

10. Bea F, Hudson FN, Neff-Laford H, White CC, Kavanagh TJ, Kreuzer J, Preusch MR, Blessing E, Katus HA and Rosenfeld ME: Homocysteine stimulates antioxidant response element-mediated expression of glutamate-cysteine ligase in mouse macrophages. Atherosclerosis 203: 105-111, 2009.

11. Lu SC: Regulation of glutathione synthesis. Mol Aspects Med 30: 42-59, 2009.

12. Lu SC: Glutathione synthesis. Biochim Biophys Acta 1830: 3143-3153, 2013.

13. Niu LY, Guan YS, Chen YZ, Wu LZ, Tung CH and Yang QZ: A turn-on fluorescent sensor for the discrimination of cystein from homocystein and glutathione. Chem Commun (Camb) 49: 1294-1296, 2013.

14. Soeur J, Eilstein J, Léreaux G, Jones C and Marrot L: Skin resistance to oxidative stress induced by resveratrol: From Nrf2 activation to GSH biosynthesis. Free Radic Biol Med 78: 213-223, 2015.

15. Bell KF, Fowler JH, Al-Mubarak B, Horsburgh K and Hardingham GE: Activation of Nrf2-regulated glutathione pathway genes by ischemic preconditioning. Oxid Med Cell Longev 2011: 689524, 2011.

16. Harvey CJ, Thimmulappa RK, Singh A, Blake DJ, Ling G, Wakabayashi N, Fujii J, Myers A and Biswal S: Nrf2-regulated glutathione recycling independent of biosynthesis is critical for cell survival during oxidative stress. Free Radic Biol Med 46: 443-453, 2009.

17. Ho HK, White CC, Fernandez C, Fausto N, Kavanagh TJ, Nelson SD and Bruschi SA: Nrf2 activation involves an oxidative-stress independent pathway in tetrafluoroethylcysteine-induced cytotoxicity. Toxicol Sci 86: 354-364, 2005.

18. Jayakumar S, Kunwar A, Sandur SK, Pandey BN and Chaubey RC: Differential response of DU145 and PC3 prostate cancer cells to ionizing radiation: Role of reactive oxygen species, GSH and Nrf2 in radiosensitivity. Biochim Biophys Acta 1840: 485-494, 2014.

19. Chen CC, Chen HL, Hsieh CW, Yang YL and Wung BS: Upregulation of NF-E2-related factor-2-dependent glutathione by carnosol provokes a cytoprotective response and enhances cell survival. Acta Pharmacol Sin 32: 62-69, 2011.

20. Livak KJ and Schmittgen TD: Analysis of relative gene expression data using real-time quantitative PCR and the 2(-Delta Delta C(T)) Method. Methods 25: 402-408, 2001.

21. Gong Z, Yan S, Zhang P, Huang Y and Wang L: Effects of S-adenosylmethionine on liver methionine metabolism and steatosis with ethanol-induced liver injury in rats. Hepatol Int 2: 346-352, 2008.

22. Paul R and Borah A: The potential physiological crosstalk and interrelationship between two sovereign endogenous amines, melatonin and homocysteine. Life Sci 139: 97-107, 2015.

23. Schalinske KL and Smazal AL: Homocysteine imbalance: A pathological metabolic marker. Adv Nutr 3: 755-762, 2012.

24. Medici V, Peerson JM, Stabler SP, French SW, Gregory JF III, Virata MC, Albanese A, Bowlus CL, Devaraj S, Panacek EA, et al: Impaired homocysteine transsulfuration is an indicator of alcoholic liver disease. J Hepatol 53: 551-557, 2010.

25. Steele ML, Fuller S, Patel M, Kersaitis C, Ooi L and Münch G: Effect of Nrf2 activators on release of glutathione, cysteinylglycine and homocysteine by human U373 astroglial cells. Redox Biol 1: 441-445, 2013.

26. Gong P and Cederbaum AI: Transcription factor Nrf2 protects HepG2 cells against CYP2E1 plus arachidonic acid-dependent toxicity. J Biol Chem 281: 14573-14579, 2006.

27. Pacana T, Cazanave S, Verdianelli A, Patel V, Min HK, Mirshahi F, Quinlivan E and Sanyal AJ: Dysregulated hepatic methionine metabolism drives homocysteine elevation in diet-induced nonalcoholic fatty liver disease. PLoS One 10: e0136822, 2015.

28. Zhou Y, Zhao L, Zhang Z and Lu X: Protective effect of enalapril against methionine-enriched diet-induced hypertension: Role of endoplasmic reticulum and oxidative stress. Biomed Res Int 2015: 724876, 2015. 